United Nations Educational Scientific and Cultural Organization and

International Atomic Energy Agency

THE ABDUS SALAM INTERNATIONAL CENTRE FOR THEORETICAL PHYSICS

\title{
A NEW TREND IN COMPLEX DIFFERENTIAL EQUATIONS: QUASIMEROMORPHIC SOLUTIONS
}

\author{
G. Barsegian ${ }^{1}$ \\ Institute of Mathematic National Academy of Science of Armenia, \\ Marshal Bagramian ave. 24-b, Yerevan 375019, Republic of Armenia \\ and \\ The Abdus Salam International Centre for Theoretical Physics, Trieste, Italy, \\ A. Sarkisian \\ Institute of Mathematic National Academy of Science of Armenia, \\ Marshal Bagramian ave. 24-b, Yerevan 375019, Republic of Armenia \\ and \\ C.C. Yang ${ }^{2}$ \\ Department of Mathematics, Hong Kong University of Science and Technology, \\ Kowloon, Hong Kong, People's Republic of China.
}

\begin{abstract}
Complex differential equations study analytic or meromorphic solutions of equations. We start a new trend by considering quasimeromorphic solutions for generalized algebraic differential equations of the first order. Particularly, the classical Goldberg's result that any meromorphic solution of a first order algebraic differential equation must be of finite order has been extended to any $K(r)$-quasimerormorphic (in $\mathbb{C}$ ) solutions of first order generalized algebraic differential equation.
\end{abstract}

MIRAMARE - TRIESTE

June 2002

\footnotetext{
${ }^{1}$ barseg@instmath.sci.am

${ }^{2}$ mayang@ust.hk
} 


\section{Introduction and results.}

We introduce the class of $K(r)$-quasimeromorphic functions and briefly show that the corresponding main results of the value distribution theory (Ahlfors' version) are also valid: for a full and detailed account of the value distribution theory for this class the reader is referred to [10]. In the present paper as an application of the theory we consider $K(r)$-quasimeromorphic solutions of generalized algebraic differential equations of the first order.

Complex (particularly algebraic) differential equations have been studied very intensively for their diverse applications in many other mathematical topics. One can also observe that there is a strong tendency to consider different type generalizations of analytic functions in many topics earlier connected or devoted to analytic or meromorphic functions. In the present paper we start to consider quasimeromorphic solutions of some generalizations of classes of algebraic differential equations that relate to linear or nonlinear partial differential equations.

The topic of algebraic differential equations is the most well developed part in the theory of complex differential equations. It studies differential equations of the form

$$
P\left(z, w, w^{\prime}\right):=\sum_{i=0}^{m} P_{i}(z, w)\left(w^{\prime}\right)^{m-i}=0,
$$

where $P$ is a polynomial in each of its arguments. An important part of the theory of algebraic differential equations deals with analytic or meromorphic solutions $w$ of the following system of equations

$$
P\left(z, w, w^{\prime}\right)=0, w_{\bar{z}}=0
$$

the second equation here means that $w$ is analytic (meromorphic), and

$$
w_{\bar{z}}:=\frac{1}{2}\left\{\frac{\partial w}{\partial x}+i \frac{\partial w}{\partial y}\right\}
$$

where $z=x+i y \in \mathbb{C}$.

We extend the class of solutions of the system of equations (1.1) by deleting the second condition $w_{\bar{z}}=0$ and by introducing the so-called quasimeromorphic solutions. Namely, we consider complex valued functions $f(z)$ which will be called quasimeromorphic if $f$ is twice differentiable in $x$ and $y$ and furthermore, $f$ can be expressed as $f=w(\psi)$, where $w$ is analytic and $\psi$ is a quasiconformal homeomorphism; it is just a generalization of the quasiconformal functions (see Lehto [12] chapter 6, p 239). Thus a quasimeromorphic function $f$ also means that $f$ admits a finite number of points $z_{i}$ in any finite disks $D(r):=\{z|| z \mid<r\}$, such that function $f \rightarrow \infty$ as $z \rightarrow z_{i}$ and $1 / f$ is quasiconformal in some neighborhoods of each $z_{i}$.

Also $f$ is quasimeromorphic then $f$ satisfies an equation $f_{\bar{z}}=q(z) f_{z},|q|<1$, (see [12], p. 239), where

$$
f_{z}:=\frac{1}{2}\left\{\frac{\partial f}{\partial x}-i \frac{\partial f}{\partial y}\right\}
$$


is the so-called formal (or complex) derivative which when $f$ is analytic becomes the usual derivative $f^{\prime}$. Note that, in general, a solution of equation $f_{\bar{z}}=q(z) f_{z},|q|<1$ may not be differentiable in $\mathbb{C}$.

We will consider quasimeromorphic solutions of equations

$$
F\left(x, y, \operatorname{Re} f, \operatorname{Im} f, f_{z}\right)=0,
$$

or what is the same solutions of

$$
F\left(x, y, \operatorname{Re} f, \operatorname{Im} f, f_{z}\right)=0, f_{\bar{z}}=q(x, y) f_{z},|q|<1 .
$$

Here the solutions will be $K$-quasimeromorphic if $|q(x, y)|<\frac{K-1}{K+1}<1$, with a positive number $K>1$ (see Ahlfors [1], pages 12-13).

A classical Gol'dberg's result [11] says that any solution $w(z)$ of equation (1.1), which is meromorphic in the complex plane must be of finite order. Particularly, this means that both Nevanlinna characteristic function $T(r, w)$ or Ahlfors-Simizu spherical characteristic function $A(r, w)$ (see [13]) are of finite order.

In the present paper we study the growth of quasimeromorphic solutions $f$ of equations (1.2) in terms of Ahlfors-Simizu spherical characteristic function $A(r, f)$.

We shall give upper bounds above bounds for solutions of equations (1.2) with $F\left(x, y, \operatorname{Re} f, \operatorname{Im} f, f_{z}\right)$ satisfying the following properties:

1. Instead of polynomials in (1.1) we consider functions $F\left(x, y, \operatorname{Re} f, \operatorname{Im} f, f_{z}\right)$ which only are required to be polynomial in $f_{z}$, with no restrictions to other arguments. Thus

$$
F\left(x, y, \operatorname{Re} f, \operatorname{Im} f, f_{z}\right):=\sum_{i=0}^{m} F_{i}(x, y, \operatorname{Re} f, \operatorname{Im} f)\left(f_{z}\right)^{m-i} .
$$

2. Furthermore, for functions $F_{i}(x, y, \operatorname{Re} f, \operatorname{Im} f$, ), we assume that for any given $i$ there is a finite set of complex values $a_{1}(i), a_{2}(i), \ldots, a_{N(i)}(i)$ lying in a disk $|a|<R<\infty$ and there are some positive constants $c(i), p(i)$ such that

$$
\left|F_{i}(x, y, \operatorname{Re} f, \operatorname{Im} f)\right| \leq c(i)|z|^{p(i)}, i \geq 1
$$

and

$$
\left|\frac{c(0)}{|z|^{p(0)}}\right| \leq\left|F_{0}(x, y, \operatorname{Re} f, \operatorname{Im} f)\right|, i=0,
$$

where $z:=x+i y \in\left\{z\left|w(z) \in a, \min _{1 \leq k \leq N(i)}\right| a-a_{k}(i)|>\rho| z \mid,>r_{0}=\right.$ const $\left.>0\right\}$.

Remark 1. Note that functions $F_{i}(x, y, \operatorname{Re} f, \operatorname{Im} f)$ substantially generalize the corresponding algebraic differential equations (1.1), where

$$
P_{i}(z, w):=\sum_{i=0}^{T} P_{i, j}(w)(z)^{T-j}
$$


and $P_{i, j}(w)$ are polynomials with respect to $w$. Note, that in this case for any value $w=a$ lying on a positive distance $\rho$ from zeros of polynomial $P_{i, 0}(w)$ with the first term $A_{i, 0} w^{M_{i, 0}}$ of degree $T$, we have

$$
\frac{\left|A_{i, 0}\right| \rho^{M_{i, 0}}}{2}|z|^{T} \leq\left|P_{i}(z, w)\right| \leq 2 \max _{j} \max _{|w|<R}\left|P_{i, j}(w)\right||z|^{T},
$$

for any point $z \in\left\{z|w(z) \in a| z \mid,>r_{0}=\right.$ const $\left.>0\right\}$.

Remark 2. Such function $F_{i}(x, y, \operatorname{Re} f, \operatorname{Im} f)$ can be expressed as

$$
\sum_{i=0}^{T}\left\{F_{i, j} \circ w\right\}(z)^{T-j} .
$$

where $F_{i, j}$ are arbitrary functions meromorphic in $\mathbb{C}$ and $F_{i, j} \circ w$ means superposition.

Thus it is pertinent to use the following

Definition. We say that $F\left(x, y, \operatorname{Re} f, \operatorname{Im} f, f_{z}\right)=0$ is a generalized algebraic differential equation if $F(\ldots$.$) satisfies (1.3),(1,4)$ and $(1.5)$.

Theorem 1. Any $K$-quasimeromorphic solution $f$ in $\mathbb{C}$ of a generalized algebraic differential equation is of finite order $\rho(f)$.

Immediately, according to Remark 2 we have

Corollary 1. Let $P\left(z, w, w^{\prime}\right)$ be a polynomial with respect to variables $z, w$ and $w^{\prime}$, which has coefficients that are meromorphic functions in $\mathbb{C}$ with respect to variable $w$; that is $P\left(z, w, w^{\prime}\right)$ can be expressed as $\sum F_{\gamma}(w) z^{\alpha} w^{\beta}\left(w^{\prime}\right)^{\gamma}$. Then any meromorphic solution (in $\mathbb{C}$ ) $w$ of the equation $P\left(z, w, w^{\prime}\right)=0$ is of finite order $\rho(f)$.

Note that the equation in Corollary 1 becomes an ordinary algebraic differential equation if $F_{\gamma}(w)$ are some constants.

An example. The follwoing is an example of such a generalized equation, which admits a solution $w=\exp z$ :

$$
\left(w^{\prime}\right)^{2} \cos ^{2} w+(w)^{2} \sin ^{2} w-w^{\prime} w=0
$$

Corollary 2 (Gol'dberg, [11]). Any meromorphic solution (in $\mathbb{C}$ ) of a first order algebraic differential equation must be of finite order.

Consider function $f$ in $\mathbb{C}$ which is $K(r)$-quasimeromorphic in the disks $\{z|| z \mid<r\}$ for all $r>r_{0}$, where

$$
K(r) \leq A^{1-3 \varepsilon}(r), \varepsilon=\text { const }, 0<\varepsilon<1 / 6 .
$$


It is easy to see that Theorem 1 immediately follows from the following more general result, which deals with $K(r)$-quasimeromorphic solutions.

Theorem 2. Any $K(r)$-quasimeromorphic (in $\mathbb{C}$ ) solution of generalized algebraic differential equation is of finite order.

Further we will consider equations involving not only $f_{z}$ but also $f_{\bar{z}}$. Let $T\left(z, f_{z}, f_{\bar{z}}\right)$ be a polynomial with respect to variables $z, f_{z}, f_{\bar{z}}$ such that for a given quasimeromorphic function $f$ in $\mathbb{C}$. Then there exists a polynomial $P(z)$ and number $p, 0 \leq p<\infty$ such that

$$
\left|f_{z}\right| \leq|P(z)|\left|T\left(z, f_{z}, f_{\bar{z}}\right)\right|^{p}
$$

for all $\left\{z|| z \mid>r^{\prime}=\right.$ const $>0,\left|f_{z}\right|>R^{\prime}=$ const $\left.>0\right\}$. As an example we can take, for instance, $T\left(z, f_{z}, f_{\bar{z}}\right):=P_{1}(z) P_{2}\left(f_{z}\right)-P_{3}(z) P_{4}\left(f_{\bar{z}}\right)$, where $P_{j}$ are polynomials with degrees $p_{1}>p_{3}$ and $p_{2}>p_{4}$. We can also consider equations with numerous other conditions, say, taking into account, in the previous formula not only degrees but also coefficients of polynomials. Substituting in $F\left(x, y, \operatorname{Re} f, \operatorname{Im} f, f_{z}\right)$ the variable $f_{z}$ by $T\left(z, f_{z}, f_{\bar{z}}\right)$, we obtain another type of generalized algebraic differential equation

$$
F\left(x, y, \operatorname{Re} f, \operatorname{Im} f, T\left(z, f_{z}, f_{\bar{z}}\right)\right)=0
$$

where it is assumed that the corresponding coefficients satisfy (1.3), (1.4) and (1.5).

Theorem 3. Let $T\left(z, f_{z}, f_{\bar{z}}\right)$ be a polynomial satisfying (1.7) and $f$ be a $K$-quasimeromorphic function that satisfies generalized algebraic differential equation (1.8) and conditions (1.3), (1.4) and (1.5). Then $f$ is of finite order.

It follows from Theorem 3 that Theorem 2 holds by taking $\left.T\left(z, f_{z}, f_{\bar{z}}\right)\right) \equiv f_{z}, P(z) \equiv 1$, $p=1$ and note (1.7).

2. Lemmas and proofs of the results. We shall use the theory of covering surfaces developed by L. Ahlfors and the method of estimating derivatives [2] to prove our results. Ahlfors theory was first applied to algebraic differential equations in [3], by combining it with the so-called proximity property of a-points, the method of estimating derivatives was derived [2], which easily led to some new types of problems and generalizations. For instance, a new stability phenomenon was studied in [4] and geometric locations of $a$-point of solutions of equations in [5], as well as new generalizations and further applications of the method can be found in [6], [7] and [8].

Classical Ahlfors' theory of covering surfaces, gives a metric-topological approach to the main conclusions of the Nevanlinna theory. A great advantage of this theory is that it enables one 
to extend easily the main conclusions of the Nevanlinna value distribution theory to the more general classes of $K$-quasimeromorphic functions $f$ in $\mathbb{C}$, which is what we will prove next.

We note here the connection between main magnitudes $A(r):=A(r, f)$ and $L(r):=L(r, f)$, where $\pi A(r)$ is the spherical area of $f(\{z|| z \mid<r\}), L(r)$ is the spherical length of boundary of $f(\{z|| z \mid<r\})$, which plays a crucial role in Ahlfors' theory of covering surfaces.

Lemma 1 (Ahlfors, [13], Chap. 13]). Let $f$ be a $K$-quasimeromorphic function in $\mathbb{C}$. Then for arbitrary $\varepsilon, 0<\varepsilon<1 / 2$, is valid

$$
L(r) \leq A^{(1-2 \varepsilon) / 2}(r)
$$

for $r \notin E_{K}$, where $E_{K}$ is a set of finite logarithmic measure.

It follows from (2.1) that

$$
\liminf _{r \rightarrow \infty} \frac{L(r)}{A(r)}=0 .
$$

The above condition is called regular exhaustion. It appears that for function satisfying the condition above, main conclusions of value distribution theory can be derived from Ahlfors' theory.

By adopting Ahlfors' arguments we easily conclude now that the condition of regular exhaustion is fulfilled for much larger classes of functions.

Note that Ahlfors derives (2.1) from the following inequality

$$
\frac{1}{r} \leq 4 \pi K \frac{d A(r)}{L^{2}(r)}
$$

Now assume that function $f$ is $K(r)$-quasimeromorphic in all the disks $\{z|| z \mid<r\}$ for all $r>r_{0}$, where $K(r) \leq A^{1-3 \varepsilon}(r), \varepsilon$ is a constant, $0<\varepsilon<1 / 6$. Denote by $E_{K(r)}$ the set of values $r>r_{0}$ for which $L(r) \geq A^{1-\varepsilon}(r)$. Then by following Ahlfors' idea, by integrating (2.3) over the set $E_{K(r)}$ of values $r$ such that $L(r) \geq A^{1-\varepsilon}(r)$, we have

$$
\begin{aligned}
\int_{E_{K(r)}} d \log r \leq 4 \pi & \int_{E_{K(r)}} \frac{K(r) d A(r)}{L^{2}(r)} \leq 4 \pi \int_{E_{K(r)}} \frac{A^{1-3 \varepsilon}(r) d A(r)}{A^{2-2 \varepsilon}(r)} \\
& \leq 4 \pi \int_{E_{K(r)}} \frac{d t}{t^{t+\varepsilon}} \leq \frac{4 \pi}{\varepsilon} 4 \pi \frac{1}{r_{0}^{\varepsilon}}
\end{aligned}
$$

which means that the set $E_{K(r)}$ is of finite logarithmic measure.

Consequently $L(r) \leq A^{1-\varepsilon}(r)$, if $r \notin E_{K(r)}$. Thus we have

Lemma 2. Let $f$ be a $K(r)$-quasiconformal function in $\mathbb{C}$. Then for arbitrary $\varepsilon, 0<\varepsilon<1 / 6$,

$$
L(r) \leq A^{1-\varepsilon}(r)
$$

for $r \notin E_{K(r)}$, where $E_{K(r)}$ is a set of finite logarithmic measure. 
Lemma 2 means that all $K(r)$-quasiconformal functions $f$ are regular exhausting.

For further applications, we need the first main theorem of Ahlfors' theory in some special particular case. Let $F_{r}$ be the image of $\{z|| z \mid<r\}$ under mapping by $K$-quasimeromorphic function $f$; and let $F_{r}^{*}$ be spherical image of $F_{r}$ on the Riemann sphere. Let $D$ be a domain on the Riemann sphere; $J_{0}(D)$ be the spherical area of $D ; J(r, D)$ be the sum of areas of all parts of $F_{r}^{*}$ lying over $D$. The magnitude

$$
s(r, D):=\frac{J(r, D)}{J_{0}(D)}
$$

is called "averaged number of sheets of the surface $F_{r}^{*}$ lying over $D$ ".

Lemma 3. First Fundamental Theorem of Ahlfors, [13]. Let $f$ be a K-quasimeromorphic function in $\mathbb{C}$. Then

$$
|s(r, D)-A(r)| \leq h L(r),
$$

where $|h|<h(D)=$ const.

From Lemmas 1-3 we have the following key lemma:

Lemma 4. Let $f$ be a $K$-quasimeromorphic function in $\mathbb{C}$. Then for arbitrary constant $\varepsilon$, $0<\varepsilon<1 / 2$,

$$
|s(r, D)-A(r)| \leq h A^{\frac{1}{2}-\varepsilon}(r),
$$

where $|h|<h(D)=$ const and $r \notin E_{K}$, where $E_{K}$ is a set of finite logarithmic measure.

Let $f$ be a $K(r)$-quasimeromorphic function in $\mathbb{C}$. Then for arbitrary $\varepsilon, 0<\varepsilon<1 / 6$,

$$
|s(r, D)-A(r)| \leq h A^{1-\varepsilon}(r),
$$

where $|h|<h(D)=$ const and $r \notin E_{K(r)}$, where $E_{K(r)}$ is a set of finite logarithmic measure.

We will apply Lemma 4 to prove Theorem 2. To this end, recall first that all roots of an algebraic equation

$$
z^{m}+b_{1} z^{m-1}+\ldots+b_{m}=0
$$

lie in the disk $|z| \leq M:=\max _{1 \leq i \leq m}\left(m\left|b_{i}\right|\right)^{1 / i}$, see [14]. Applying this to equation (1.2) we get for any value $z$

$$
\left|f_{z}(z)\right| \leq \max _{1 \leq i \leq m}\left(m\left|\frac{F_{i}(x, y, \operatorname{Re} f, \operatorname{Im} f)}{F_{0}(x, y, \operatorname{Re} f, \operatorname{Im} f)}\right|\right)^{1 / i} .
$$

We will choose now a disk $D \subset\{a|| a \mid<R\},(R$ is as defined in Remark 1$)$, such that the distances $d$ between $D$ and all the points $a_{1}(i), a_{2}(i), \ldots, a_{N(i)}(i), i=0,1,2, \ldots, m$, are positive. For all values $a \in D$ we consider their preimages $z_{i}(a)$, so that $f\left(z_{i}(a)\right)=a$. By taking into account (1.4), (1.5), (2.6) and (2.7) we have that for any $z_{i}(a),\left|z_{i}(a)\right|>r_{0}$,

$$
\left|f_{z}(z)\right| \leq \max _{1 \leq i \leq m}\left(m\left|\frac{c(i)|z|^{p(i)+p(0)}}{c(0)}\right|\right)^{1 / i} .
$$


Since, for the Jacobian $J(z)$ of function $f$,

$$
J(z):=\left|f_{z}\right|^{2}-\left|f_{\bar{z}}\right|^{2} \leq\left|f_{z}\right|^{2}
$$

we conclude that the element of the spherical area of mapping $f$ is $J(z) /\left(1+|w(z)|^{2}\right)^{2}$ and thus

$$
A(r, f):=\frac{1}{\pi} \iint_{|z|<r} \frac{J(z)}{\left(1+|f(z)|^{2}\right)^{2}} d \sigma
$$

where, $d \sigma$ is the Euclidean element of area, and

$$
s(r, D, f):=\iint_{z(r, D)} \frac{J(z)}{\left(1+|f(z)|^{2}\right)^{2}} d \sigma
$$

where $z(r, D):=\{z|| z \mid<r, f(z) \in D\}$.

Now, taking into account (2.8) and (2.9), we have

$$
\begin{gathered}
s(r, D, f):=s\left(r_{0}, D, f\right)+\iint_{z(r, D) \backslash z\left(r_{0}, D\right)} \frac{J(z)}{\left(1+|f(z)|^{2}\right)^{2}} d \sigma \leq \frac{1}{J(D)} A\left(r_{0}, f\right)+ \\
+\max _{\{z|| z \mid<r\}} \max _{1 \leq i \leq m}\left(m\left|\frac{c(i)|z|^{p(i)+p(0)}}{c(0)}\right|\right)^{2 / i} \iint_{z(r, D) \backslash z\left(r_{0}, D\right)} \frac{1}{\left(1+|f(z)|^{2}\right)^{2}} d \sigma \leq \\
\leq \frac{1}{J(D)} A\left(r_{0}, f\right)+\pi r^{2} \max _{\{z|| z \mid<r\}} \max _{1 \leq i \leq m}\left(m\left|\frac{c(i)|z|^{p(i)+p(0)}}{c(0)}\right|\right)^{2 / i} \leq \\
\leq \pi\left|\frac{m}{c(0)}\right|^{2 / i} r^{2(p(i)+p(0)) / i+2} .
\end{gathered}
$$

Now starting with a value $r^{*}>r_{0}$, we have

$$
s(r, D, f) \leq 2 \pi\left|\frac{m}{c(0)}\right|^{2 / i} r^{2(p(i)+p(0)) / i+2}
$$

and by inequality (2.6) of Lemma 4

$$
A(r) \leq 2 \pi\left|\frac{m}{c(0)}\right|^{2 / i} r^{2(p(i)+p(0)) / i+2}+h A^{1-\varepsilon}(r) .
$$

Finally, we conclude that starting with a new value $r^{* *}>r^{*}$ depending only on $f, h$ and $\varepsilon$, the following inequality holds:

$$
A(r) \leq 3 \pi\left|\frac{m}{c(0)}\right|^{2 / i} r^{2(p(i)+p(0)) / i+2},
$$

for $r \notin E_{K(r)} \backslash\left[0, r^{* *}\right)$. Since the characteristic function $A(r)$ is monotonically increasing and $E_{K(r)}$ has a finite logarithmic measure, it follows that for all values $r$ greater than the value $r^{* * *}>r^{* *}$,

$$
\begin{aligned}
A(r) & \leq 3 \pi\left|\frac{m}{c(0)}\right|^{2 / i}(2 r)^{2(p(i)+p(0)) / i+2} \\
& \leq\left\{3 \pi\left|\frac{m}{c(0)}\right|^{2 / i} 2^{2(p(i)+p(0)+\varepsilon) / i+2}\right\} r^{2(p(i)+p(0)) / i+2}
\end{aligned}
$$


which also completes the proof of Theorem 2 .

To prove Theorem 3 it is enough to note that, by arguing similarly as in the proof of inequality (2.7), we can get the upper bounds for $|T|$ and then, due to (1.7), we can get some upper bounds for $\left|f_{z}\right|$. Then Theorem 3 follows by repeating the arguments in the proof of Theorem 2 .

Examples of equations with quasimeromorphic solutions. Let $T$ be an affine nondegenerating transformation that can be expressed in complex form as

$$
T:=T(w):=U+i V=\{a u+b v\}+i\{c u+d v\}, /: /: w=u+i v
$$

Denote its inverse transformation by $T^{-1}$, that is

$$
T^{-1}:=u+i v=\left\{a^{\prime} U+b^{\prime} V\right\}+i\left\{c^{\prime} U+d^{\prime} V\right\}
$$

and by $T^{*}(w)$ transformation

$$
\{(a-d) u+(b+c) v\}+i\{(c-b) u+(d+a) v\}
$$

Note that if $w=u+i v$ is a meromorphic function, then $T(w)$ is quasimeromorphic function $f:=U+i V$. Calculating formal derivative for $T(w)$, we have

$$
f_{z}=\left\{(a-d) \frac{\partial u}{\partial x}+(b+c) \frac{\partial v}{\partial x}\right\}+i\left\{(c-b) \frac{\partial u}{\partial x}+(d+a) \frac{\partial v}{\partial x}\right\}=T^{*}\left(w^{\prime}\right) .
$$

Now let $w$ denote a meromorphic (in $\mathbb{C}$ ) solution of the equation:

$$
w^{\prime}=P_{1}(z) w^{2}+P_{2}(z) w+P_{3}(z)
$$

where $P_{1}(z), P_{2}(z), P_{3}(z)$ are polynomials. By applying transformation $T^{*}$ to both sides of (2.11) and by $(2.10)$, we obtain

$$
f_{z}=T^{*}\left(P_{1}(z) w^{2}+P_{2}(z) w+P_{3}(z)\right)
$$

where the right-hand-side can be rewritten as a function $F^{*}(x, y, u, v)$. In turn, applying transformation $T^{-1}$ we can rewrite $F^{*}(x, y, u, v)$ as $F^{* *}(x, y, U, V)$. Thus function $f=U+i V$ is a quasimeromorphic solution of the following equation

$$
f_{z}=F^{* *}(x, y, U, V)
$$

It is easy to check that for function

$$
F\left(x, y, \operatorname{Re} f, \operatorname{Im} f, f_{z}\right):=f_{z}-F^{* *}(x, y, U, V)
$$

conditions (1.3), (1.4) and (1.5) of Theorem 1 are fulfilled.

Concluding Remark. In fact, above we have essentially proved that the main results of the classical value distribution theory that describe $a$-points are also true for $K(r)$-quasiconformal 
functions $f$ in $\mathbb{C}$. Indeed, Ahlfors' theory gives analogues of the first and second fundamental theorems of Nevanlinna for any class of "smmoth enough" functions that satisfy the regular exhaustion condition. Moreover, the class mentioned in his theory is the class of $K$-quasimeromorphic functions. Due to Lemma 4, $K(r)$-quasiconformal functions $f$ in $\mathbb{C}$ are also regular exhausting, therefor, for them, the main conclusions mentioned are true. Further, a proximity property of $a$-points [8], mentioned above, generalize the main conclusions of value distributions theory by describing not only numbers of $a$-points but also their geometric locations. Again this proxomity property is true, see proofs in [9], for any class of "smooth enough" and regular exhausting functions. Consequently, it will be true for $K(r)$-quasiconformal functions $f$ in $\mathbb{C}$ as well.

Acknowledgements. The first author would like to thank the Abdus Salam International Centre for Theoretical Physics (ICTP), Trieste, Italy, for kind hospitality and support.

\section{References}

[1]. Ahlfors L., Lectures on quasiconformal mappings, Van Nostrand Company, Inc. Princeton, New Jersey, 1966.

[2]. Barsegian G., Estimates of derivative of meromorphic functions oh sets of $a$-points, Journal of London Math. Soc., 34, n.2, 1986, pp. 534-540.

[3]. Barsegian G., Applications of L. Ahlfors theory of covering surfaces to the study of algebraic differential equations of the second orders, Differentsialnye Uravnenija, v.23, n. 2, 1987, pp. 341344.

[4]. Barsegian G., Laine I. and Yang C.C., Stability phenomenon and problems for complex differential equations with relations to sharing values, Mathematichni Studii, v. 13, n. 2, pp. 224-228.

[5]. Barsegian G., Laine, Yang C.C., Locations of values of meromorphic solutions of algebraic differential equations, Complex Variables, v. 46, 2001, pp. 323 - 336.

[6]. Barsegian G., Begehr H. and Laine I., Stability phenomenon for generalizations of algebraic differential equations, Zeitshirft fur. Analysis und ihre Anwendungen, 21, n. 2, (2002), pp. 495 -503 .

[7]. Barsegian G., Laine I. and Yang C. C., On a method of estimating derivatives in complex differential equations, Journal of Japan Math. Soc. (to appear)

[8]. Barsegian G.A. and Yang C.C., On the cross-road of Value distribution $\Gamma$-lines, Free Boundary theories and applied mathematics, Preprint.

[9]. Barsegian G., Proximity property of $a$-points of meromorphic functions, Math. Sbornic, v. 120(162), n. 1, 1983, pp. 42-63, (in Russian); Transl. in "Math. USSR Sbornik".

[10]. Barsegian G., Sarkisian A., and Yang C.C., Value distribution of $K(r)$-quasimeromorphic function and applications to differential equations, Preprint. 
[11]. Goldberg A.A., On integrals of differential equations of the first order, Ukrainskij Matem.Journal, (in Russian), 8, (1956), pp. 254-261.

[12]. Lehto O., Virtatnen K.I., Quasiconformal mappings in the plane, Springer-Veralg, Berlin, Heidelberg, New York, 1973.

[13]. Nevanlinna R., Eindeutige analytische Funktionen, Springer, 1936.

[14]. Polya G. and Szegő, Problems and theorems from analysis. vol. 2, Berlin. Springer, 1954. 